Research Article

\title{
Neuromodulatory Effect of Sensorimotor Network Functional Connectivity of Temporal Three-Needle Therapy for Ischemic Stroke Patients with Motor Dysfunction: Study Protocol for a Randomized, Patient-Assessor Blind, Controlled, Neuroimaging Trial
}

\author{
Ning Zhao ${ }^{1},{ }^{1,2}$ Hong Zhang, ${ }^{3}$ Tongyan Liu, ${ }^{3}$ Jiao Liu, ${ }^{1}$ Yun Xiang, ${ }^{2}$ Guojian Shu, ${ }^{2}$ \\ Chunzhen $\mathrm{Li}^{2}{ }^{2}$ Jingwen Xie, ${ }^{2}$ and Lidian Chen $\mathbb{D}^{1}$ \\ ${ }^{1}$ Fujian University of Traditional Chinese Medicine, Fuzhou 350122, China \\ ${ }^{2}$ The 6th Affiliated Hospital of Shenzhen University Health Science Center, Shenzhen Nanshan District People's Hospital, \\ Shenzhen 518052, China \\ ${ }^{3}$ Hunan University of Traditional Chinese Medicine, Changsha 410007, China
}

Correspondence should be addressed to Lidian Chen; cld@fjtcm.edu.cn

Received 13 August 2020; Revised 8 October 2020; Accepted 13 November 2020; Published 4 January 2021

Academic Editor: Hai-dong Guo

Copyright (c) 2021 Ning Zhao et al. This is an open access article distributed under the Creative Commons Attribution License, which permits unrestricted use, distribution, and reproduction in any medium, provided the original work is properly cited.

Background. The clinical efficacy of temporal three-needle therapy for stroke dysfunction has been previously demonstrated in China. However, the central mechanism of temporal three-needle therapy remains unclear. Temporal three-needle projects the sensory cortex and the motor cortex, which may impact the cortex function. Current studies seldom focus on it. Hence, according to the "scalp-cortex corresponding theory," the underlying mechanism of temporal three-needle remains a domain for further research. Methods. This trial is designed to provide objective and visual evidence for the neuromodulatory effect and neuroimaging mechanism of temporal three-needle therapy for stroke patients. This ongoing study is a prospective, randomized, controlled, patient-assessor blind, single-center, neuroimaging trial involving two-parallel patient groups and a healthy control group. Forty eligible patients will be recruited from Shenzhen Nanshan District People's Hospital and randomized into either the experimental group or the control group. Twenty healthy volunteers will be recruited in the healthy control group and undergo baseline magnetic resonance imaging scans without any intervention. Patients in the control group will receive acupuncture at Dingnieqianxiexian (MS6), in addition to basic medicine and rehabilitative treatments. Patients in the experimental group will receive temporal three-needle therapy plus basic medicine and rehabilitative treatments 5 days per week, 10 sessions over two consecutive weeks. The primary outcome is resting-state functional connectivity, and the secondary outcomes are regional homogeneity, amplitude of low-frequency fluctuations, Fugl-Meyer assessment of the upper limb, and modified Barthel Index. All outcome measures will be assessed at baseline and after 2 weeks of intervention. Discussion. The results will explore the neuromodulatory effects and illustrate the central mechanism of temporal three-needle treatment from the network-level viewpoint of sensorimotor network functional plasticity and promote widespread application in real-world practice. This trial was registered at Chinese Clinical Trial Registry on 14 March 2018 with ChiCTR1800015209.

\section{Introduction}

Stroke is the second leading cause of death, and Kim et al reported that stroke remained the third-leading cause of yearsof-potential-life lost worldwide [1]. The stroke incidence ranged from 76 per 100,000 population per year up to 119 per 100,000 population per year in different countries [2], and it is expected that the incidence of stroke will continue to increase as the population ages [3]. There are about two million new patients of stroke annually in China [4]. Most of the stroke survivors are 
associated with functional disabilities in China [5], and motor dysfunction is the most significant symptom, which results in different levels of dysfunction (including muscle atrophy, joint stiffness, shoulder pain, deep vein thrombosis of the lower limb, long-term brakes, infection, anxiety, and depression) and disability in daily life. The stroke impact in China is more severe compared to the worldwide average levels. DALYs (disability adjusted life-years) caused by stroke rank 3rd in global epidemiologic study and first in China [6]. Overall poststroke care costs were the highest in the USA ( $\$ 4850 /$ per patient month) [7], which indicates that stroke not only brings pain to patients themselves but also imposes a great financial burden on families and communities in most countries, which has become a critical public health problem.

In 2002, the World Health Organization recommended acupuncture as a therapy for stroke [8]. Temporal threeneedle acupuncture is one of Jin's three-needle therapy systems created by Professor Jinrui in China. The temporal I needle is located on the hairline 2 cun above the apex of the ear; the temporal II needle and the temporal III needle are located 1 cun from the temporal I needle on either side in the horizontal direction [9]. Temporal three-needle therapy integrates the meridian acupoint theory in Chinese traditional medicine, modern neurological theory, and anatomical rationale $[10,11]$. The location of the temporal three-needle is highly overlapped with the temporal bone seams, which is a crevice left by the congenital development process [12]. Additionally, the nerve is extended into the skull through the gap of the skull, which can effectively mobilize the neuron activity after acupuncture, and the position of the temporal three-needle ranged including the precentral gyrus and postcentral gyrus could widely affect the motor and sensory areas [13]. Meanwhile, the bioelectric effect of acupuncture at the location can be transmitted to the cerebral cortex, which can quickly rebuild the function of injured neurons in the functional area and strengthen the compensatory ability of the brain [11]. Moreover, the temporal bone is much thinner than other skulls, and its suture is mostly densest; acupuncture sensation is easier to be conducted, and the acupuncture effect is better. As a result, the acupoint selection of temporal three-needle has a certain representative. In real-world clinical practice, acupuncture on the left temporal three-needle treats right limb paralysis and acupuncture on the right temporal threeneedle for the left paralysis, which is widely used in the poststroke rehabilitation in China [11, 14-18], especially motor dysfunction $[14,15,19]$.

Because the study design is a superiority trial and stroke patients conventionally receive acupuncture at the scalp acupoint in current clinical practice in China, placebo (sham) stimulation or a blank control may violate the patient willingness. This trial will choose Dingnieqianxiexian (MS6) in the International Standardization Scheme for Scalp Acupuncture Points as the positive comparator in the control group. MS6 is an international standardized scalp acupoint, which refers to a diagonal line from Qianshencong (EX-HN1) to Xuanli (GB6) [20], and is projected to the precentral gyrus [21]; it is responsible for the autonomous movement of the limbs [20]. Studies have indicated that, on the basis of body acupuncture, temporal three-needle therapy is superior to acupuncture at MS6 with respect to daily life activities [22], motor function [22], and motorrelated sensory disturbances [19].

However, the central mechanism of temporal threeneedle therapy on stroke recovery remains unclear, especially in neuroimaging. From the last several years, with the rapid development of medical imaging technology, magnetic resonance imaging (MRI) has gradually become one of the main methods for studying the mechanism of acupuncture. Resting-state fMRI (rs-fMRI) only requires the subjects to stay still and close their eyes while scanning and keep their body as immobile as possible without thinking about anything. Rs-fMRI reflects intrinsic functional patterns and the spontaneous activity of the brain's cortex [23], which is a useful technique for studying the mechanism of acupuncture [24]. In this trial, we plan to apply rs-fMRI to explore the central mechanism of temporal three-needle treatment.

Previous neuroimaging studies demonstrated that motor disorders in stroke patients were associated with functional connectivity (FC) abnormalities in the sensorimotor network or sensorimotor cortex $[25,26]$. Moreover, functional recovery after stroke is associated with preserved FC of motor to nonmotor networks [27]. Acupuncture can increase the default mode network and sensorimotor network functional connectivity with pain-, affective-, and memory-related brain areas compared with sham acupuncture [24]. Several studies have also shown that acupuncture increases the sensorimotor network connectivity with pain-related brain regions in healthy adults or inhibits neuroinflammation in the sensorimotor cortex after ischemic stroke $[28,29]$. However, the neuroimaging mechanism based on temporal three-needle therapy in hemiplegic patients with stroke remains unclear. The theoretical basis of scalp acupuncture is mainly based on the two aspects [20]: (1) the traditional Zangfu meridians theory and (2) scalpcortex corresponding theory. According to the "scalp-cortex corresponding theory," the temporal three-needle projects the cortex of the sensory area and the motor area on the temporal ear, and the bioelectric effect produced by acupuncture in this area is transmitted to the cerebral cortex, which can impact the function of the cerebral cortex [11]. Given the abovementioned viewpoints, we hypothesize that the underlying neuroimaging mechanism on neuromodulatory effects in temporal threeneedle acupuncture may be related to sensorimotor network functional connectivity.

Therefore, our first objective is to assess the neuromodulatory effect of temporal three-needle therapy on resting-state regional brain activity, whole-brain activity, and motor function. Our second objective is to explore the relationship between alterations in functional connectivity at the sensorimotor network level and changes in behavioral performances to demonstrate the underlying neuroimaging mechanism of temporal three-needle treatment.

\section{Methods}

2.1. Study Design and Setting. This study will be a two-week, prospective, randomized, patient-assessor blind, controlled, single-center, superiority, neuroimaging trial with two- 
parallel patient groups and a healthy control group. This study will take place mainly in Shenzhen Nanshan District People's Hospital, Shenzhen, Guangdong Province, China. In this trial, we will recruit subjects into two categories. The first category will comprise healthy controls, and the second will comprise patient subjects. A total of 20 age-, gender-, and education-matched healthy subjects will be recruited into the healthy control group. The purpose of healthy control recruitment is to establish a baseline normal MRI data set to facilitate comparison with the MRI data of the patients' groups. The healthy control group will receive baseline MRI scans without any intervention. Forty eligible patients will be randomized into two groups: (1) the experimental group and (2) the control group, with a $1: 1$ allocation ratio. All patients will take medicine for secondary prevention of stroke (e.g., antihypertensive, hypoglycemic, antiplatelet, and hypolipidemic drugs) and conventional rehabilitative treatments according to the Chinese stroke rehabilitation treatment guidelines [5] (e.g., physical therapy (PT) and occupational therapy (OT)); these treatments will be consistent across the patient groups. In the control group, patients will receive acupuncture at MS6 following basic drug treatments and conventional rehabilitative treatments. Patients in the experimental group will receive temporal three-needle acupuncture 5 days per week, 10 sessions over 2 consecutive weeks, plus basic drug treatments and conventional rehabilitative treatments. Acupuncturists with at least 10 years of clinical experience will perform acupuncture therapy (including temporal three-needle therapy and MS6 therapy). Professional rehabilitative therapists with above 5 years of clinical experience will conduct conventional rehabilitative treatments (PT and OT) for the patients.

The planned flowchart of the trial is shown in Figure 1. The timeline for assessment is provided in Table 1. This study protocol is in compliance with the requirements of the Helsinki Declaration. Ethical approval was provided by the Ethics Committee of Shenzhen Nanshan District People's Hospital in February 2018. In addition, Shenzhen Nanshan District People's Hospital is also carrying out this trial and is responsible for the coordination of all departments' activities (e.g., study protocol registry, staff training, informed consent implementation, and data management). This protocol follows Standard Protocol Item: Recommendations for Interventional Trials (SPIRIT) 2013 [30] (see Additional File 1 in Supplementary Materials available here) and the Consolidated Standards of Reporting Trials (CONSORT) 2010 statement for nonpharmacological interventions [31].

2.2. Sample Size Calculation. Our primary outcome will be resting-state FC measured by rs-fMRI. Because millions of voxels are used to indirectly estimate the blood oxygen leveldependent (BOLD) signal and traditional power calculations are of no significance [32], we calculated the sample size according to the relevant published articles. Previous rsfMRI study of the acupuncture mechanisms in stroke had a sample size of 20 cases for the healthy control group [33]. Moreover, a study on the effects of sample size on cerebral response to acupuncture with fMRI had shown that the imaging results in 17 healthy controls were similar to the results of studies with 21 cases [34], which may suggest that approximately 20 subjects maybe a relatively common choice in rs-fMRI studies after acupuncture. The sample size in acupuncture mechanism study using rs-fMRI with stroke patients typically ranges from a few cases to approximately 20 cases [33, 35-37]. Because this trial is a neuroimaging study, the primary outcome will be based on the subjects' MRI image processing, and patients with left cerebral ischemia accompanied by right limb hemiplegia will be enrolled in this study so as to ensure the relative consistency of cerebral ischemia lesions and reveal the neuroimaging mechanism more regularly.

Under the condition of left brain ischemia stroke with right limb hemiplegia in this study, we plan to enroll 20 patients diagnosed with ischemia stroke in each patient group (20 in the experimental group and 20 in the control group) and 20 healthy volunteers in the healthy control group.

2.3. Participants and Recruitment. Forty patients diagnosed with stroke will be recruited from Shenzhen Nanshan District People's Hospital. Three trial staff will conduct the recruitment in accordance with the inclusion and exclusion criteria to determine whether the patients are eligible for the study. Patients will be recruited through oral recruitment during daily consultation, advertisements, WeChat in Shenzhen, and volunteer recommendations. All patients' subjects will undergo a baseline evaluation, including complete the collection of demographic and clinical data.

After completion of the recruitment of the forty patients, a total of 20 age-, gender-, and education-matched healthy subjects will be recruited from the community-dwelling population in the Nanshan District in Shenzhen, Guangdong Province, China, according to the demographic characteristics of the experimental group. The recruitment methods will be the same as that of the patients.

Additionally, all patients and healthy controls will be invited to voluntarily sign the informed consent form.

2.4. Diagnostic Criteria. The diagnostic criteria for ischemic stroke (cerebral infarction) are as follows [38]: (1) most acute onset occurs in the static state, as dynamic onset patients are more common in cardiogenic cerebral infarction, and some cases may have transient cerebral ischemia attacks before onset; (2) aggravation or fluctuation is present; (3) clinical manifestations depend on the size and location of the infarct focus, mainly the symptoms and signs of focal neurological deficits, such as hemiplegia, paresthesia, aphasia, and ataxia, with some patients having headaches and vomiting, in coma, and others with whole-brain symptoms; and (4) there is confirmation via brain CT or MRI.

2.4.1. Inclusion Criteria. The inclusion criteria for patients with stroke are as follows: (1) diagnosis of ischemic stroke confirmed by brain CT or MRI; (2) left cerebral ischemia (including the stroke patients with anterior and posterior 


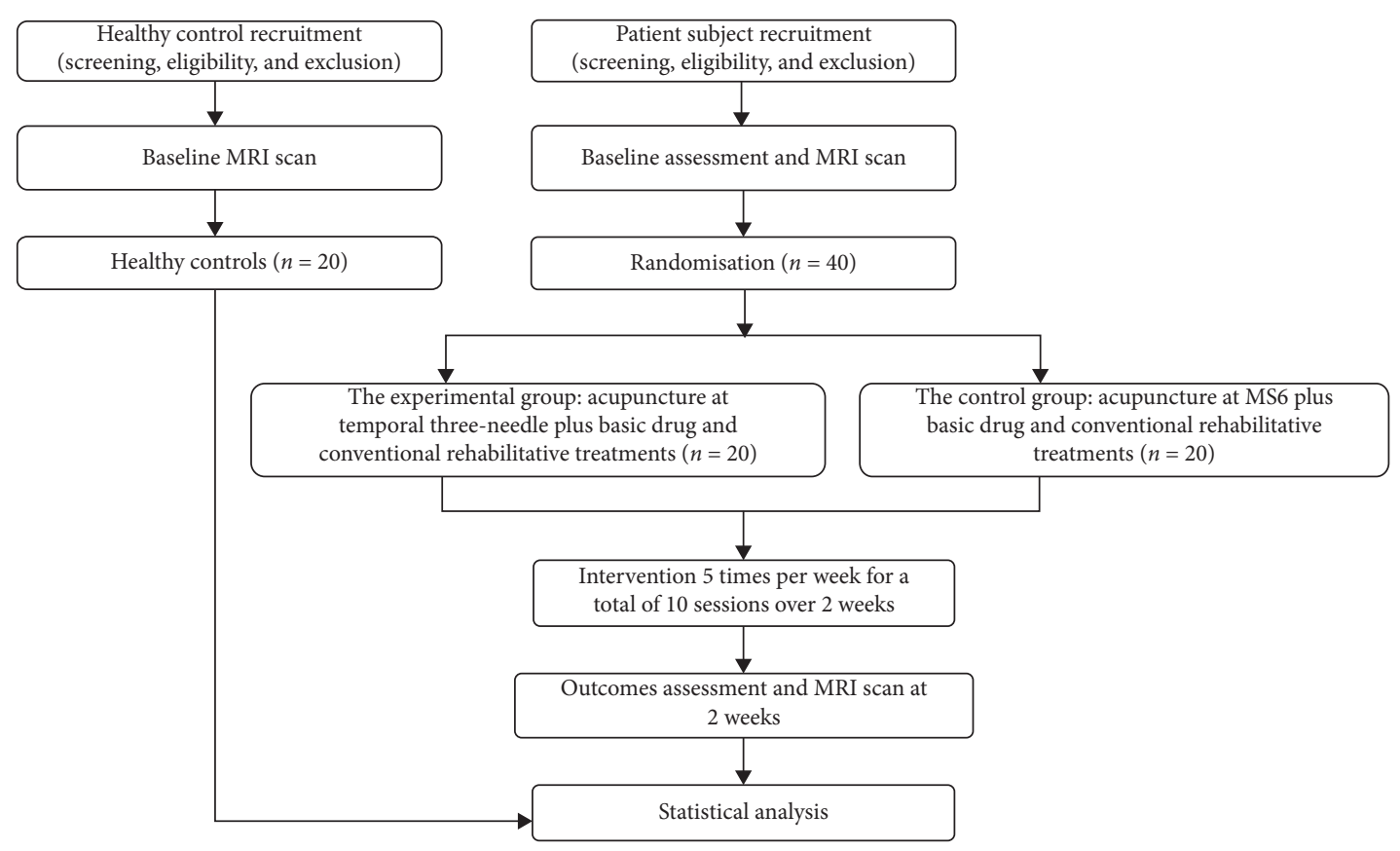

FIgURe 1: Planned flowchart of the trial.

TABLE 1: Study schedule for enrollment, interventions, and assessments.

\begin{tabular}{|c|c|c|c|c|}
\hline \multicolumn{5}{|c|}{ Study period } \\
\hline \multirow{2}{*}{$\frac{\text { Items }}{\text { Time points }}$} & \multicolumn{2}{|c|}{ Baseline } & \multirow{2}{*}{$\begin{array}{c}\text { Treatment phase } \\
1-2 \text { weeks }\end{array}$} & \multirow{2}{*}{$\frac{\text { Outcomes assessment }}{3 \text { weeks }}$} \\
\hline & -1 to -3 days & 0 weeks & & \\
\hline \multicolumn{5}{|l|}{ Enrollment } \\
\hline Eligibility screen & $x$ & & & \\
\hline Informed consent & $x$ & & & \\
\hline Examination & & $\times$ & & \\
\hline Randomization & & $\times$ & & \\
\hline \multicolumn{5}{|l|}{ Intervention } \\
\hline \multicolumn{5}{|l|}{ Healthy control group $(n=20)$} \\
\hline Experimental group $(n=20)$ & & & $x$ & \\
\hline Control group $(n=20)$ & & & $x$ & \\
\hline \multicolumn{5}{|l|}{ MRI scan } \\
\hline Healthy control group & & $x$ & & \\
\hline Experimental group & & $\times$ & & $\times$ \\
\hline Control group & & $x$ & & $\times$ \\
\hline \multicolumn{5}{|c|}{ Assessment (only in the experimental group and the control group) } \\
\hline FMA & & $x$ & & $x$ \\
\hline MBI & & $x$ & & $\times$ \\
\hline \multicolumn{5}{|l|}{ Safety } \\
\hline Adverse events & & & $x$ & $\times$ \\
\hline
\end{tabular}

circulation infarction) with right limb hemiplegia; (3) firstever clinical stroke occurring between 2 weeks and six months; (4) aged between 30 and 70 years; (5) stable medical condition, Brunnstrom stages I-V; and (6) written informed consent to participate.

The inclusion criteria for healthy controls include the following: (1) can be matched to the patient's age, gender, and education; (2) has no acupuncture treatment in the last month; (3) has no history of head trauma, psychiatric disease, neurological disease, or physical activity disorder; (4) has no MRI contradictions; and (5) has provided written informed consent.
2.4.2. Exclusion Criteria. The exclusion criteria are as follows: (1) a history of previous traumatic brain injury, tumor, or epilepsy; (2) bilateral hemiplegia; (3) critical organ failure, such as heart, lung, liver, and kidney failure; (4) severe cognitive impairment and inability to cooperate; (5) claustrophobia, wearing a pacemaker, intracranial metal implants, or skull defects; (6) severe cervical lesions including severe cervical spinal stenosis and cervical instability; (7) women who are pregnant or lactating; (8) contradictions for MRI scans; (9) currently participation in another clinical trial that would affect the evaluation results 
of this study; or (10) fear of needling, needle phobia, and fainting with needles.

2.4.3. Dropout Criteria. During the trial period, participants meeting the following criteria will be excluded from the study: (1) occurrence of serious adverse events; (2) missing more than 3 of 10 acupuncture treatment sessions; (3) important violations in the protocol implementation; or (4) withdrawal from the study in light of the participants being unwilling to continue.

2.5. Randomization and Allocation. After meeting the selection criteria, 40 eligible patients will be randomly assigned into either the experimental group or the control group at an allocation ratio of $1: 1$ after signing written informed consent forms. The random sequence will be created by a thirdparty professional statistician of Shenzhen University using STATA 12.0 software (StataCorp, Texas, USA). The randomization list will only be seen by this research coordinator and will be concealed from other study personnel. Participant assignments will be hidden using sequentially numbered, opaque, sealed envelopes. The research coordinator that assigns the groups will not participate in the patients' inclusion, the subsequent trial process, or the assessment of results.

2.6. Blinding. The trial will be conducted using a patientassessor blind design. The patients, assessors, and statisticians will not be aware of which group the patients are in. They will only be told whether data will belong to group A or group B, but they will not be aware of which group is the experimental group or the control group. Furthermore, they will not even know whether the trial is designed to be a noninferiority trial, an equivalent trial, or a superiority trial, thus avoiding human factors affecting the results of the data analysis.

Regarding acupuncture implementation, acupuncture doctors cannot be blinded, so unblinded acupuncture doctors will apply the acupuncture intervention depending on the patients' intervention arm.

The blind method will be applied throughout the whole study process. A member of the trial staff will supervise the process of blind implementation and will disclose the blind code after the statistical analysis is completed. Unblinding will be considered only in a critical medical emergency.

\subsection{Treatment}

2.7.1. Basic Drug Treatment. Combined with their past stroke-related risk factors and current medical histories, all patients will receive secondary prophylactic drug treatment for stroke, including antihypertensive, hypoglycemic, antiplatelet, and hypolipidemic drugs.

\subsubsection{Conventional Rehabilitative Interventions.} Conventional rehabilitation treatment will be administered 5 days per week, 10 sessions ( 2 consecutive weeks): (a) Physical therapy [5]: PT will include active and passive activity of affected limb joint, neurodevelopmental therapy, balance function training, antispasmodic treatment, muscle strength training, and walking gait training.

(b) Occupational therapy [5]: OT will include therapeutic activities for the upper limbs and hands of the affected side, activities of daily living, and training for the use of auxiliary equipment.

PT and OT will be performed by professional physiotherapists with at least 5 years of clinical experience. Each patient's pre- and postrehabilitative intervention will be conducted by the same physiotherapist.

2.7.3. Experimental Group. The experimental group will receive acupuncture at temporal three-needle 5 times per week for 2 weeks following basic drug treatment and conventional rehabilitative treatment. The details of temporal three-needle therapy are presented in Table 2; the therapy will comply with the Standards for Reporting Interventions in Clinical Trials of Acupuncture (STRICTA) checklist [39].

The temporal I needle will be placed on the hairline 2 cun above the apex of the ear; the temporal II needle and temporal III needle will be placed 1 cun from the temporal I needle on either side in the horizontal direction [13] (Figure 2; the use of the picture has been allowed by the author Tongyan Liu and the patient). After routine disinfection of the scalp, disposable stainless-steel needles $(0.30 \times 25 \mathrm{~mm})$ (Huanqiu, Suzhou Acupuncture \& Moxibustion Appliance Co., Ltd., Jiangsu Province, China) will be manually inserted at each acupoint. After the acupuncturists insert needles into the acupoints at a certain depth and twist them for a while, patients report conscious sensations, such as soreness and numbness, in the acupuncture location, which indicates needle sensation "De Qi." To treat motor dysfunction, the needles are rotated at 100 revolutions or more per minute for 1 minute at every 5 minutes over 30 minutes.

2.7.4. Control Group. The control group will receive acupuncture at the MS6 from Qianshencong (EX-HN1) to Xuanli (GB6) [20] following basic drug treatments and conventional rehabilitative treatments. The details of acupuncture at MS6 are presented in Table 2; this treatment will comply with the STRICTA checklist [39] as well.

2.8. Outcome Assessment. The outcome measurements include functional connectivity (FC), regional homogeneity (ReHo), amplitude of low-frequency fluctuations (ALFF), the Fugl-Meyer assessment of upper limb (FMA-UL), the modified Barthel Index (MBI), and adverse events. FC, ReHo, and ALFF will be measured by rs-fMRI. The FMAUL, MBI, and adverse events (AEs) will be assessed by three experienced rehabilitative assessors.

Additionally, the study duration will be 2 weeks. Outcomes will be assessed at baseline and after 2 weeks of 
TABLE 2: STRICTA checklist (details of intervention).

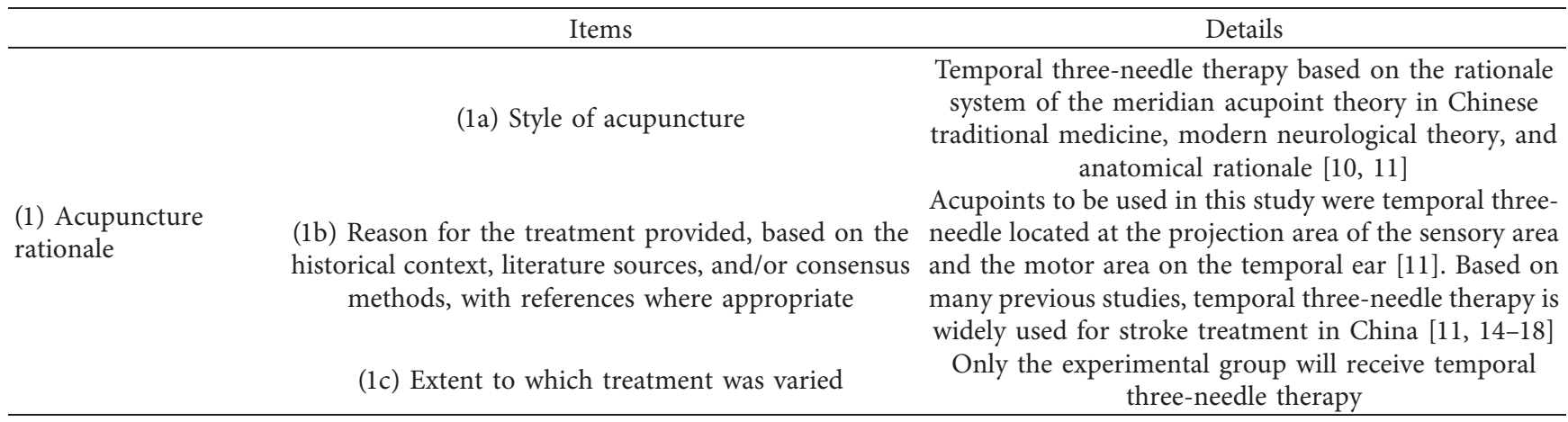

(2a) Number of needle insertions per subject per session

3

(2b) Names of points used

Temporal three-needle

(2c) Depth of insertion, based on a specified unit of measurement

(2) Details of needling (2d) Response sought

(2e) Needle stimulation

(2f) Needle retention time

(2g) Needle type

\section{5 inches deep}

"De Qi" sensation

Manual operation

30 minutes

Sterilized stainless-steel needle measuring $0.30 \times 25 \mathrm{~mm}$

(Huanqiu, Suzhou Acupuncture \& Moxibustion

Appliance Co., Ltd, Jiangsu Province, China)

(3a) Numbers of treatment sessions (3b) Frequency and duration of treatment sessions

(4a) Details of other interventions administered to the acupuncture group

(4) Other components of treatment

(4b) Setting and context of treatment, including instructions to practitioners and information and explanations to patients

(5) Practitioner background

(5) Description of participating acupuncturists

10 sessions ( 2 consecutive weeks) 30 minutes per day, 5 days per week

All patients will receive basic drugs treatment and conventional rehabilitative treatments (including PT and OT)

The study will be conducted in Shenzhen Nanshan district People's Hospital, and all information will be provided to the patients

Acupuncturists after completing 5 years of Chinese medicine undergraduate course with more than 10 years of clinical experience will perform the temporal threeneedle interventions

(6a) Rationale for the control or comparator in the context of the research question, with sources that justify this choice

(6) Control or comparator interventions (6b) Precise description of the control or comparator; if sham acupuncture or any other types of acupuncturelike control is used, provide details as for items 1 to 3 above
MS6 will be selected as the comparator in the control group based on traditional Chinese medicine theory

Based on previous studies [19, 22], MS6 will be mainly selected as the comparator for temporal three-needle therapy in motor dysfunction with stroke patients; in addition to the basic drug treatments and conventional rehabilitative treatment, only the control group will receive MS6 therapy; item 2 (details of needling) and item 3 (treatment regimen) are the same as the experimental group intervention. Each subject will have the same assessor for their pre- and posttreatment evaluations.

2.8.1. Primary Outcome. The primary outcome is restingstate FC. Functional brain alterations in this study will be more sensitive than structural MRI and behavioral performances in detecting the neuromodulatory effect of temporal three-needle therapy.

Resting-state FC analyses can assess synchronous activity between brain regions and be used to study the brain in healthy and disease groups on the brain-network level, which will help to understand (a) markers for diseases, (b) paths to investigate disease mechanisms, and (c) predictors of clinical course [40]. Resting-state FC could serve as a biomarker of motor function recovery in stroke patients with hemiplegia [41]. Stronger FC after stroke correlates with better behavioral outcome [42]. Resting-state FC analysis may offer an invaluable approach to explore functional network reorganization in the brain after stroke. In this study, brain FC plasticity within and beyond the sensorimotor network will be measured by rs-fMRI.

2.8.2. Secondary Outcomes. The secondary outcomes including ReHo, ALFF, FMA-UL, and MBI will be measured at 


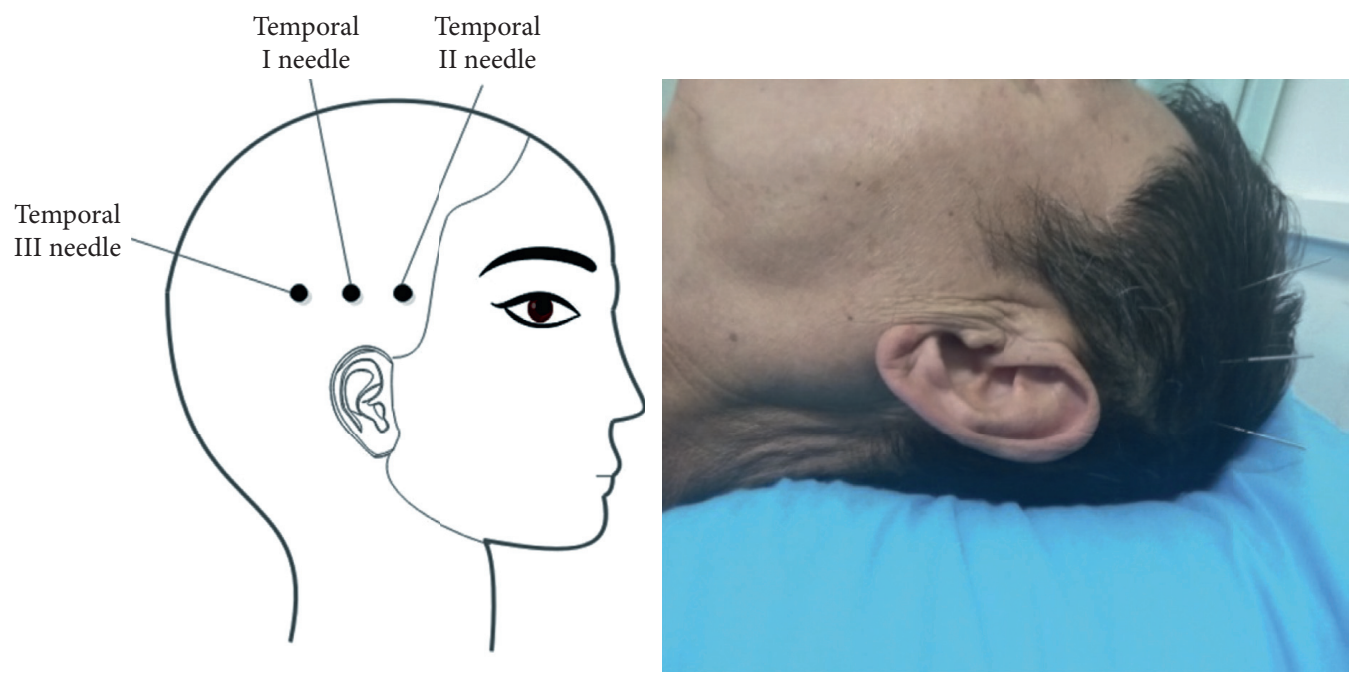

Figure 2: Locations of temporal three-needle therapy in the experimental group (the use of the picture has been allowed by the author Tongyan Liu and the patient).

the same time points of the primary outcome. Brain regional activity (ReHo and ALFF) will be assessed via rs-fMRI. FMA-UL, MBI, and AEs will be assessed by the abovementioned three experience rehabilitative assessors.

ReHo can be used to explore the consistency of neuronal activity in regional brain areas at resting state, which may be a potential biomarker of the neural substrates associated with hand function following stroke [43] and provide an effective tool for evaluating the efficiency of rehabilitative therapies after stroke [44]. An increase in ReHo value indicates that the consistency of neuron activity tends to be increased.

ALFF can reflect the intensity of the nerve activity of a single voxel and hemodynamic BOLD information on neural activity [45]. In addition to FC, regional ALFF may provide complementary information on the neural mechanism [46]. After brain injury, the blood flow of the brain decreases, and the local ALFF value shows a downward trend.

The FMA-UL is used to only assess motor function of the upper limb in stroke patients. Because the motor function of the upper limb is the most difficult to recover after stroke, we focused on upper limb motor dysfunction in this study. The FMA-UL has been shown to be useful for measuring arm and hand motor function, and 30-item assessment shows a longitudinally stable item difficulty order and is valid for measuring volitional arm motor ability over time [47]. The FMA has a total of 100 points for normal motor function. The maximum score for UL is 66. The evaluation includes measuring reflex activity, flexor synergy, extensor synergy, and movement combining synergies, movement out of synergy, elbow, shoulder, wrist, and hand function, and relates coordination and speed. An ordinal scale applied to each item is used: $0=$ cannot be performed, $1=$ can be partially performed, and $2=$ can be completely performed.

The MBI has shown good properties for measuring disability in stroke patients [48] and is the most prevalent functional outcome scale in stroke trials. The MBI covers 10 domains: feeding, grooming, dressing, bathing, toilet transfers, bowel and bladder continence, ambulation, moving between wheel chair bed and back to the wheel chair, and stair climbing. Each item has five levels, with level 5 representing the greatest independence.

2.9. Brain MRI Protocol. Healthy controls will undergo a baseline brain MRI scan, and patients in the experimental group and the control group will undergo brain MRI scans at baseline and after treatment. All brain MRI scans will be performed with a 3.0 Tesla scanner (SIEMENS Skyra, Germany) in the Department of Radiology, Shenzhen Nanshan District People's Hospital. The parameters of the sequences were as follows.

Structure MRI: prior to brain functional imaging scan, T1-weighted images of three-dimensional whole-brain structure in sagittal position will be collected using MPRAGE sequence with the following parameters: repetition time (TR)/echo time $(\mathrm{TE})=2530 / 2.01 \mathrm{~ms}$, flip angle $(\mathrm{FA})=7^{\circ}$, field of view $(\mathrm{FOV})=256 \times 256 \mathrm{~mm}^{2}$, matrix $=256 \times 256,176$ contiguous axial slices, slice spacing $=0.0 \mathrm{~mm}$, and slice thickness $=1.0 \mathrm{~mm}$.

T2 FLAIR (fluid-attenuated inversion recovery):T2 FLAIR will be collected using a turbo spin echo (TSE) sequence with the following parameters: $T R=8500 \mathrm{~ms}$, $\mathrm{TE}=102 \mathrm{~ms}$, and flip angle $=160^{\circ}$.

Rs-fMRI: resting-state functional images will be performed using an echo planar imaging (EPI) sequence with the following parameters: $\mathrm{TR} / \mathrm{TE}=2000 / 30 \mathrm{~ms}, \mathrm{FA}=90^{\circ}$, number of $\quad$ slices $=36, \quad$ matrix $=64 \times 64$, $\mathrm{FOV}=224 \times 224 \mathrm{~mm}^{2}$, and slice thickness $=3.5 \mathrm{~mm}$.

2.10. Data Collection, Management, and Monitoring. The design, modification, and final confirmation of the case report form (CRF) will be jointly conducted by Shenzhen Nanshan District People's Hospital and researchers. The CRF must ultimately be approved by Shenzhen Nanshan 
District People's Hospital. Electronic CRF will be utilized for data collection. The data entry will be performed separately by two independent staff.

Specialized data manager will be used to maintain the integrity, validity, and correctness of the data and to fully check the primary and secondary outcomes and security indicators specified in the plan to ensure the accuracy and completeness of these data.

Shenzhen Nanshan District People's Hospital is responsible for data collection, data entry, data verification and query, medical coding, blind audit, data export and transmission, archiving of data, and data management.

\subsection{Statistical Analysis}

2.11.1. MRI Data Analysis. The DPABI toolkit [49] will be used to analyze the image data on MATLAB2016 (MathWorks) to detect any functional changes (FC, ReHo, and ALFF) in brain neuroplasticity due to temporal three-needle treatment. After data preprocessing, data-driven approach will be conducted to explore neuroplastic changes in the two patient groups and the healthy control group. Data-driven approaches can automatically obtain the brain functional network from the data [50]. Independent component analysis (ICA) based on data-driven approaches is not required to assume a priori that the MRI signal can be separated directly into a series of maximally independent components and associated time courses $[51,52]$ and different components corresponding to the corresponding resting-state network. FDR correction will be used for multiple comparisons to control the false positivity rate. Pearson's correlation analysis will be utilized to study the relationship between the rs-fMRI metrics and behavioral data.

2.11.2. Clinical Behavioral Data Analysis. In this study, statistical analyses will be performed by intent-to-treat (ITT) analysis and per-protocol (PP) analysis. The ITT analysis will include not only all patients with valid results but also randomized patients who were discontinued for a period of time for some reason or transferred to another group. For missing values, we will carry over the last observation results to the endpoint so that the number of patients at the endpoint of each group will be the same as that at the beginning of the trial. The PP analysis will be applied to subjects who adhere to the protocol, have good compliance, and complete the process. When differences in the results exist between the two analyses, the final clinical trial results will be discussed and explained. In this study, only those who received more than $70 \%$ of the interventions will be considered satisfied compliance. That is to say, in total number of the 10 treatments, subject missing more than 3 treatments will be excluded from the PP analysis due to violation of the protocol.

All differences in the baseline demographic and clinical characteristics between the two patient groups will be analyzed with the chi-squared test or Fisher's exact test for categorical data and with the $t$-test or Wilcoxon rank-sum test for continuous values. Continuous variables will be shown as the means, SDs. Categorical variables will be described as frequencies or percentages.

All tests will be two-sided, and a $p$ value $<0.05$ will be considered statistically significant. If the difference of the primary endpoint between the experimental group and the control group was statistically significant, the results of the experimental group are superior to that of the control group, and the intervention method could be considered as effective. Therefore, we firstly set the type I error of the primary endpoint at 0.05 . If significant, we then go to test the secondary endpoint at 0.05 type I error.

The changes in the FMA and the MBI between pre- and postacupuncture therapy will be analyzed using repeated measures analysis of variance (ANOVA), This analysis will be used to compare differences and to examine the group effect, time effect, and time-group interaction effect.

A post hoc test will be conducted. Within-group differences between pre- and posttreatment will be compared using the paired $t$-test or Wilcoxon signed rank test for continuous data.

AEs will be tabulated and summarized using descriptive statistics. The AEs incidence of each group will be confirmed as evaluation index, and the computation formula is as follows: AEs incidence $\%=$ (number of AEs/total number of cases in this group) $\times 100 \%$. The current version of STATA 12.0 statistics (StataCorp, Texas, USA) will be used for statistical analyses.

2.12. Quality Control. Shenzhen Nanshan District People's Hospital will monitor the following aspects of the trial regularly and strictly: the division and training for the research staff, file management, informed consent, protocol compliance, subject recruitment, filling in the CRF, intervention, quality assured system, statistical analysis, and data management.

2.13. Investigation of Adverse Events and Safety. Intervention-related AEs might include edema due to bleeding, bending and breaking of the needle, fainting during acupuncture, and retained needle after treatment. During the course of the study, any AEs including the onset of clinical symptoms, degree of symptom severity, persistence time, treatment, and sequelae will be recorded. AEs will be compared between patients in the experimental group and the control group and will be recorded in the CRF and assessed by the research staff at each visit. Serious AEs will be reported to the ethics committee immediately.

2.14. Trial Status. Currently, this trial is at the recruitment phase with some subjects being involved.

\section{Discussion}

The similarities between the temporal three-needle and MS6 are as follows: (1) the two are both locating on the side of the skull and (2) the temporal three-needle and MS6 can treat contralateral central motor dysfunction $[11,20]$. The 
differences between the temporal three-needle and MS6 are as follows: (1) the acupoint projecting brain regions are different: the temporal three-needle projects the sensory region and the motor region including the precentral gyrus and the postcentral gyrus, which may be responsible for sensory function and motor function of the limbs [13], and MS6 projects the precentral gyrus [21], which manages autonomous limb movement [20]; (2) the acupoint-related meridians are different: the temporal three-needle connects the Gallbladder Meridian of Foot-Shaoyang (GB), Bladder Meridian of Foot-Taiyang (BL), Stomach Meridian of FootYangming (ST), and Sanjiao Meridian of Hand-Shaoyang (SJ); MS6 connects the Du meridian (DU), BL, and GB; (3) the acupoint stimulation sensation is different: the temporal three-needle is located at the temporal sutures which have a high degree overlap, and the gaps left by this congenital development process can help the needle sensation of the extracranial scalp penetrate deeper into the skull $[10,11,13]$; however, the MS6 stimulation location includes the parietal bone, frontal bone, and temporal bone, which are not the thinnest part of the skull, so the transmission of the acupuncture sensation may be weaker than that of the temporal three-needle. In summary, because of its different projected cortex, diverse-related meridian system, deeper stimulation sensation, and wider dysfunctional treatment (the temporal three-needle focuses on both motor dysfunction and sensory dysfunction), temporal three-needle therapy is now more widely used for ischemic stroke in China.

Studies have found that abnormal brain structures and functions exist in patients after stroke compared with ageand gender-matched healthy controls $[53,54]$. In this trial, we will aim to investigate the neuromodulatory effect of sensorimotor network FC of temporal three-needle therapy for ischemic stroke patients with motor dysfunction. First, we will compare the differences in baseline rs-fMRI metrics between the healthy subjects and the stroke patients to confirm the difference between the healthy subjects and stroke patients. Second, to determine the difference due to the temporal three-needle treatment, we will compare the changes in the rs-fMRI metrics between the experimental group and the control group to demonstrate whether motor function alteration is related to brain functional neuroplastic changes after temporal three-needle treatment. Although a large number of studies on the use of temporal three-needle therapy for the treatment of motor dysfunction, speech disorders, dysphagia, and depression after stroke have indicated clinical behavioral effects $[11,14-18]$, to the best of our knowledge, our study will be the first randomized neuroimaging trial with both a positive control group and a healthy control group to examine the actual effects by means of rs-fMRI.

This study has several strengths. First, the implementation of rigorous randomized, patient-assessor blind, controlled, neuroimaging trial will provide visual and objective evidence about how temporal three-needle treatment improves motor dysfunction in ischemic stroke patients. Second, the combined assessments of rs-fMRI and behavioral data will be used to identify the neuromodulatory effects of temporal three-needle therapy in stroke patients. Third, research based on the FC within the sensorimotor network, and between the sensorimotor network and nonmotor network, is better for understanding the mechanism of temporal three-needle therapy in stroke according to the "scalp-cortex corresponding theory."

Some limitations of this study should be noted. First, the major limitation of this protocol is its lack of long-term follow-up assessments. Second, there is no sham acupuncture or blank control comparison group for observation. Third, this trial will be conducted in a single center. Fourth, only the patients of left cerebral ischemia with right limb hemiplegia will be recruited in this protocol, and the patients of right cerebral ischemia with left limb hemiplegia will not be temporarily considered enrolled in this trial. We plan to resolve the aforementioned limitations in the future.

In conclusion, the results of this study are expected to explore the neuromodulatory effects and characterize the central mechanism of temporal three-needle treatment on motor dysfunction in hemiplegic stroke patients from the networklevel viewpoint of sensorimotor network functional plasticity and to promote widespread application in real-world practice.

\section{Abbreviations \\ MRI: Magnetic resonance imaging \\ fMRI: Functional magnetic resonance imaging \\ Rs- Resting-state functional magnetic resonance \\ fMRI: imaging \\ FC: $\quad$ Functional connectivity \\ ReHo: Regional homogeneity \\ ALFF: Amplitude of low-frequency fluctuations \\ FMA: Fugl-Meyer assessment \\ MBI: $\quad$ Modified Barthel index \\ CRF: $\quad$ Case report form.}

\section{Data Availability}

Data and materials are available upon reasonable request from the co-first authors.

\section{Additional Points}

Recruitment began in March 2018 (Protocol version 1.0).

\section{Ethical Approval}

The study followed Helsinki Declarations. Ethical approval has already been obtained from the Ethics Committee of Shenzhen Nanshan District People's Hospital (Approval no. 2018-0210-1).

\section{Consent}

All participants will understand the trial procedures and the trials' purpose and potential benefits before they provide informed written consent. 


\section{Disclosure}

Ning Zhao and Hong Zhang are co-first authors. The funding body had no role in the study design or the decision to submit the manuscript for publication.

\section{Conflicts of Interest}

The authors declare that they have no conflicts of interest.

\section{Authors' Contributions}

Ning Zhao and Hong Zhang contributed equally to this work. NZ, HZ, and TYL drafted this manuscript. NZ supervised the blind implementation. TYL contributed the recruitment of the subjects. YX, GGS, and CZL contributed to outcomes assessment. JWX contributed to choosing the neuroimaging methods and conducting the statistical analyses. HZ, JL, and LDC revised the manuscript. LDC guided the protocol. All authors read and approved the final manuscript.

\section{Acknowledgments}

This research was supported by the Medical Scientific Research Foundation of Guangdong Province (Grant no. A2018551) and the Scientific Research Project of the Shenzhen Health and Family Planning System (Grant no. SZXJ2018026). The authors thank Jiaqing Liu and Binghong Duan for contributing to data entry, Li Wen and Yajie Yang for recruitment of subjects, Huagao Wang and Lu Zhang for PT, and Huixiang Xiao and Yihong Gong for OT.

\section{Supplementary Materials}

SPIRIT2013 checklist: recommended items to address in a clinical trial protocol and related documents. (Supplementary Materials)

\section{References}

[1] A. S. Kim, E. Cahill, and N. T. Cheng, "Global stroke belt," Stroke, vol. 46, no. 12, pp. 3564-3570, 2015.

[2] A. G. Thrift, T. Thayabaranathan, G. Howard et al., "Global stroke statistics," International Journal of Stroke, vol. 12, no. 1, pp. 13-32, 2017.

[3] M. Katan and A. Luft, "Global burden of stroke," Seminars in Neurology, vol. 38, no. 2, pp. 208-211, 2018.

[4] S. Wu, B. Wu, M. Liu et al., "Stroke in China: advances and challenges in epidemiology, prevention, and management," The Lancet Neurology, vol. 18, no. 4, pp. 394-405, 2019.

[5] Neurological Rehabilitation Group of Chinese Society of Neurology, Chinese Stroke Society, Office of stroke Screening and Prevention Engineering Committee, Ministry of Health, "Chinese stroke rehabilitation treatment guidelines(2011complete version)" Chinese Journal of Rehabilitation Theory and Practice, vol. 18, no. 4, pp. 301-318, 2012, in Chinese.

[6] Y. Gao, B. Jiang, H. Sun, X. Ru, D. Sun, and L. Wang, "The burden of stroke in China: results from a nationwide population-based epidemiological survey," PLoS One, vol. 13, no. 12, Article ID e0208398, 2018.
[7] S. Rajsic, H. Gothe, H. H. Borba et al., "Economic burden of stroke: a systematic review on post-stroke care," The European Journal of Health Economics, vol. 20, no. 1, pp. 107-134, 2019.

[8] WHO, Acupuncture: Review and Analysis of Reports on Controlled Clinical Trials, World Health Organization, Geneva, Switzerland, 2002.

[9] P. ZF, Jin's Three-Needle Therapy, Shanghai Scientific and Technological Literature Press, Shanghai, China, 2000, in Chinese.

[10] Y. Wei, "Jin Rui professor acupuncture therapy characteristics," Traditional Chinese Medicine Journal, vol. 1, no. 1, pp. 39-40, 2002, in Chinese.

[11] H. Dexiong, J. Chao, Z. Huanhuan, and Zhuanglixing, "Analysis of the therapeutic effect of temporal three-needle on stroke," Liaoning Journal of Traditional Chinese Medicine, vol. 36, no. 12, pp. 2064-2065, 2009, in Chinese.

[12] G. Qi and X. Xiaohu, "The important position of temporal three-needle in scalp acupuncture in children with cerebral palsy," Jiangxi Journal of Traditional Chinese Medicine, vol. 46 , no. 1 , p. 61,2015 , in Chinese.

[13] C. Tiequ, Jin Three-Needle Clinical Matched Acupoints Method, People's Medical Publishing House, Beijing, China, 2008, in Chinese.

[14] Y. Di and R. Jin, "Clinical study of temporal three-needle therapy for acute stroke," Journal of Clinical Acupuncture and Moxibustion, vol. 8, pp. 25-27, 1995, in Chinese.

[15] R. Jin, X. Lai, Y. Li, and R. Liang, "Clinical observation on temporal three-needle acupuncture therapy of sequela of apoplexy," Zhongguo Zhen Jiu, vol. 1, pp. 11-12, 1993, in Chinese.

[16] P. H. Yuan, H. X. Jun, and Z. M. Hua, "Effects on activities of daily life and quality of life of post-stroke depression treated with electroacupuncture at temple-three-needle," World Journal of Acupuncture-Moxibustion, vol. 20, no. 4, pp. 9-12, 2010.

[17] L. Tongyan, Z. Ning, L. Jiaqing et al., "Clinical observation on tongue three-needle combined with temporal three-needle in patients with dysphagia after stroke," Journal of Hunan University of Chinese Medicine, vol. 39, no. 4, pp. 516-519, 2019, in Chinese.

[18] J. Yu, S. Liu, and F. Chen, "Clinical observation on treating aphasia after stroke by acupuncture plus speech function rehabilitation," Clinical Journal of Chinese Medicine, vol. 9, no. 2, pp. 76-77, 2017, in Chinese.

[19] X. Luo, "Clinical research on therapeutic effect of paralysis after a stroke treated by acupuncuture of the scalp and body," Master's thesis, Guangzhou University of Traditional Chinese Medicine, Guangzhou, China, 2015.

[20] S. Guojie, Acupuncture for TCM. Professional Use of Integrated Traditional Chinese and Western Medicine, Shanghai Scientific and Technical Publishers, Shanghai, China, 1997, in Chinese.

[21] J. Shang and Y. Li, "Study on the relationship between scalp acupuncture acupoint distribution and cortical projected region-MS6 and MS7," Information on Traditional Chinese Medicine, vol. 2, pp. 41-42, 1987, in Chinese.

[22] H. Sheng-yang, The clinical study of temporal three-needle therapy to ischemic stroke hemiplegia patients, $\mathrm{Ph} . \mathrm{D}$. thesis, Guangzhou University of Traditional Chinese Medicine, Guangzhou, China, 2010.

[23] W. Grodd and C. F. Beckmann, "Resting state functional MRI of the brain," Nervenarzt, vol. 85, no. 6, pp. 690-700, 2014.

[24] R.-L. Cai, G.-M. Shen, H. Wang, and Y.-Y. Guan, "Brain functional connectivity network studies of acupuncture: a 
systematic review on resting-state fMRI," Journal of Integrative Medicine, vol. 16, no. 1, pp. 26-33, 2018.

[25] W. Jie, Z. Zhiyong, and T. Chaozheng, "A resting-state fMRI study of sensorimotor network in stroke based on independent component analysis," Chinese Journal of Rehabilitation Medicine, vol. 32, no. 6, pp. 607-612, 2017, in Chinese.

[26] H. Liu, T. Tian, W. Qin, K. Li, and C. Yu, "Contrasting evolutionary patterns of functional connectivity in sensorimotor and cognitive regions after stroke," Frontiers in Behavioral Neuroscience, vol. 10, p. 72, 2016.

[27] S. R. Almeida, J. Vicentini, L. Bonilha, B. M. De Campos, R. F. Casseb, and L. L. Min, "Brain connectivity and functional recovery in patients with ischemic stroke," Journal of Neuroimaging, vol. 27, no. 1, pp. 65-70, 2017.

[28] R. P. Dhond, Y. Calvin, P. Kyungmo, N. Kettner, and V. Napadow, "Acupuncture modulates resting state connectivity in default and sensorimotor brain networks," Pain, vol. 136, no. 3, pp. 407-418, 2008.

[29] W. Liu, X. Wang, S. Yang et al., "Electroacupunctre improves motor impairment via inhibition of microglia-mediated neuroinflammation in the sensorimotor cortex after ischemic stroke," Life Sciences, vol. 151, pp. 313-322, 2016.

[30] A. W. Chan, J. M. Tetzlaff, P. C. Gotzsche et al., "SPIRIT 2013 explanation and elaboration: guidance for protocols of clinical trials," BMJ, vol. 346, p. e7586, 2013.

[31] D. Moher, S. Hopewell, K. F. Schulz et al., "CONSORT 2010 explanation and elaboration: updated guidelines for reporting parallel group randomised trials," BMJ, vol. 340, p. c869, 2010.

[32] L. Jiang, H. Geng, M. Lu et al., "Acupuncture for poststroke hemiplegia focusing on cerebral bilateral connections: study protocol for a randomised controlled neuroimaging trial," BMJ Open, vol. 10, no. 4, Article ID e034548, 2020.

[33] Y. Ning, K. Li, C. Fu et al., "Enhanced functional connectivity between the bilateral primary motor cortices after acupuncture at Yanglingquan (GB34) in right-hemispheric subcortical stroke patients: a resting-state fMRI study," Frontiers in Human Neuroscience, vol. 11, p. 178, 2017.

[34] X. Chunsheng, L. Chuanfu, Y. Jun et al., "Effects of sample size on cerebral response to acupuncuture with fMRI," Chinese Imaging Journal of Integrated Traditional and Western Medicine, vol. 9, no. 4, pp. 289-292, 2011, in Chinese.

[35] C. H. Fu, K. S. Li, Y. Z. Ning et al., "Altered effective connectivity of resting state networks by acupuncture stimulation in stroke patients with left hemiplegia: a multivariate granger analysis," Medicine (Baltimore), vol. 96, no. 47, p. e8897, 2017.

[36] S.-Q. Chen, D.-C. Cai, J.-X. Chen, H. Yang, and L.-S. Liu, "Altered brain regional homogeneity following contralateral acupuncture at Quchi (LI 11) and Zusanli (ST 36) in ischemic stroke patients with left hemiplegia: an fMRI study," Chinese Journal of Integrative Medicine, vol. 26, no. 1, pp. 20-25, 2020.

[37] Y. Li, Y. Wang, C. Liao, W. Huang, and P. Wu, "Longitudinal brain functional connectivity changes of the cortical motorrelated network in subcortical stroke patients with acupuncture treatment," Neural Plasticity, vol. 2017, Article ID 5816263, 9 pages, 2017.

[38] M. L. Rao, China Guideline for Cerebrovascular Disease Prevention and Treatment, People's Medical Publishing House, Beijing, China, 2007, in Chinese.

[39] H. Macpherson, D. G. Altman, R. Hammerschlag et al., "Revised standards for reporting interventions in clinical trials of acupuncture (STRICTA): extending the CONSORT statement," PLoS Medicine, vol. 7, no. 6, Article ID e1000261, 2010.
[40] M. Ernst, S. Torrisi, N. Balderston, C. Grillon, and E. A. Hale, "fMRI functional connectivity applied to adolescent neurodevelopment," Annual Review of Clinical Psychology, vol. 11, no. 1, pp. 361-377, 2015.

[41] Y. Zhang, H. Liu, L. Wang et al., "Relationship between functional connectivity and motor function assessment in stroke patients with hemiplegia: a resting-state functional MRI study," Neuroradiology, vol. 58, no. 5, pp. 503-511, 2016.

[42] C. R. Hernandez-Castillo, J. Y. Nashed, J. Fernandez-Ruiz, J. Wang, J. Gallivan, and D. J. Cook, "Increased functional connectivity after stroke correlates with behavioral scores in non-human primate model," Scientific Reports, vol. 7, no. 1, p. 6701, 2017.

[43] Z. Zhao, C. Tang, D. Yin et al., "Frequency-specific alterations of regional homogeneity in subcortical stroke patients with different outcomes in hand function," Human Brain Mapping, vol. 39, no. 11, pp. 4373-4384, 2018.

[44] D. Yin, Y. Luo, F. Song et al., "Functional reorganization associated with outcome in hand function after stroke revealed by regional homogeneity," Neuroradiology, vol. 55, no. 6, pp. 761-770, 2013.

[45] Q.-L. Yao, H.-Y. Zhang, B.-B. Nie, F. Fang, Y. Jiao, and G.-J. Teng, "MRI assessment of amplitude of low-frequency fluctuation in rat brains with acute cerebral ischemic stroke," Neuroscience Letters, vol. 509, no. 1, pp. 22-26, 2012.

[46] M. Hu, F. Ji, Z. Lu et al., "Differential amplitude of lowfrequency fluctuations in brain networks after BCI training with and without tDCS in stroke," in Proceedings of the IEEE Engineering in Medicine and Biology Society, pp. 1050-1053, Guadalajara, Mexico, July 2018.

[47] M. L. Woodbury, C. A. Velozo, L. G. Richards, P. W. Duncan, S. Studenski, and S.-M. Lai, "Longitudinal stability of the FuglMeyer assessment of the upper extremity," Archives of Physical Medicine and Rehabilitation, vol. 89, no. 8, pp. 1563-1569, 2008.

[48] C. M. Yang, Y.-C. Wang, C.-H. Lee, M.-H. Chen, and C.-L. Hsieh, "A comparison of test-retest reliability and random measurement error of the Barthel index and modified Barthel index in patients with chronic stroke," Disability and Rehabilitation, pp. 1-5, 2020, In press.

[49] C.-G. Yan, X.-D. Wang, X.-N. Zuo, and Y.-F. Zang, "DPABI: data processing \& analysis for (resting-state) brain imaging," Neuroinformatics, vol. 14, no. 3, pp. 339-351, 2016.

[50] D. Yuhui, G. Zhiguo, L. Yingjun, and F. Chen, "Review of hypothesis driven and data driven methods for brain functional network analysis," Beijing Biomedical Engineering, vol. 32, no. 3, pp. 307-311, 2013, in Chinese.

[51] M. J. McKeown, S. Makeig, G. G. Brown et al., "Analysis of fMRI data by blind separation into independent spatial components," Human Brain Mapping, vol. 6, no. 3, pp. 160-188, 1998.

[52] V. D. Calhoun, T. Adali, G. D. Pearlson, and J. J. Pekar, "Spatial and temporal independent component analysis of functional MRI data containing a pair of task-related waveforms," Human Brain Mapping, vol. 13, no. 1, pp. 43-53, 2001.

[53] M. Yang, P. Yang, Y.-S. Fan et al., "Altered structure and intrinsic functional connectivity in post-stroke aphasia," Brain Topography, vol. 31, no. 2, pp. 300-310, 2018.

[54] J. Zhang, Y. Zhang, L. Wang et al., "Disrupted structural and functional connectivity networks in ischemic stroke patients," Neuroscience, vol. 364, pp. 212-225, 2017. 